\title{
Evaluación de la formación integral escolar a través de un diseño cuasiexperimental: contribuciones desde la Educación Física Evaluation of holistic education school through a quasi-experimental design: contributions from Physical Education
}

\author{
*Nicol Pinilla Fonseca, **María Cristina Gamboa Mora, ***Mónica Morales Barrera \\ *IE San Isidoro (Colombia), Universidad de Baja California (México); **Universidad Nacional Abierta y a Distancia (Colombia), \\ ***Universidad Nacional Autónoma de México (México)
}

\begin{abstract}
Resumen. El objetivo de este estudio fue evaluar el nivel de formación integral logrado en los estudiantes de educación media desde el área de educación física con base en la valoración de sus percepciones, si bien es cierto que actualmente existen numerosos estudios relacionados con el tema se encuentra que, no se aborda la manera como se debe desarrollar la formación integral y al plantear las estrategias se traza un horizonte para su ejecución. Se utilizó un diseño cuasiexperimental, en el que se aplicó un instrumento tipo escala Likert con 57 ítems como diagnóstico, validado estadísticamente (alfa de Cronbach 0.92) y por expertos, posteriormente se implementaron estrategias pedagógicodidácticas (juegos tradicionales, expresa lo que piensas, recreo-natación, práctica en gimnasio y Munzee entre otras), encaminadas al desarrollo integral, finalmente se aplicó el instrumento como postest y se estableció la significancia de las diferencias con la prueba deWilcoxon. La muestra se configuró con 180 estudiantes de la Institución Educativa San Isidoro del municipio de Espinal, de carácter público. Los resultados de las dimensiones reflejaron cambios estadísticos significativos desde el panorama de los estudiantes, los rangos positivos son indicadores de mejor percepción sobre la formación integral desde el área de educación física. Se concluye, que la implementación de las estrategias pedagógico-didácticas desde la educación física, contribuye a la formación integral de los estudiantes, la dimensión ética y socioemocional aportan sustancialmente.

Palabras clave: formación integral, evaluación, dimensiones humanas, media vocacional, escala Likert.
\end{abstract}

\begin{abstract}
The purpose of this study was to evaluate the holistic education achieved in high school students from the area of physical education based on the assessment of their perceptions, although it is true that currently there are numerous studies related to the subject, it is found that the way in which integral training should be developed is not addressed and when proposing the strategies, a horizon is drawn for its execution. A quasi-experimental design was used, in which a Likert scale instrument was applied with 57 items as diagnosis, statistically validated (Cronbach's alpha 0.92) and by experts, later pedagogical-didactic strategies were implemented (traditional games, express what you think, recreation-swimming, gym practice and Munzee among others), aimed at integral development, finally the instrument was applied as a posttest and the significance of the differences was established with theWilcoxon test. The sample was carried out with 180 students from San Isidro school from Espinal, which is a public school. The results of the dimensions showed significant statistical changes from the students' point of view, the positive ranges are indicators regarding a better perception of the holistic education taught in PE (physical education). In conclusion, the implementation of didactic and pedagogical strategies of PE contribute to the students' holistic education, ethical and socioemotional realm, which represent a substantial contribution.
\end{abstract}

Keys Word. Integral formation, test, human dimensión, 10th and 11th grades, Likert scale.

\section{Introducción}

La formación integral es considerada como el fin de la educación en la escuela, ya que no se trata solamente de preparar en conocimientos o conceptos, sino forjar seres humanos que estén en la capacidad de vivir en comunidad, cuidar el medio ambiente, desarrollar valores, formarse no solamente en su intelecto, se trata de fomentar personas íntegras y felices. "Comprehensive training includes knowledge of the social reality surrounding these players and the opportunities to improve it» (Almorza, Pérez y Prada, 2020, p. 376), al comprender el contexto y

Fecha recepción: 06-04-21. Fecha de aceptación: 22-08-21

Nicol Pinilla Fonseca

nicolpinillafonseca@gmail.com la realidad social de los estudiantes para poder mejorarla, se está contribuyendo con un proceso de formación integral.

Es relevante visibilizar a la educación como proceso de humanización, en el cual la integralidad tiene sentido y significado para el ser humano, en la medida que permite la realización de las personas, a través de la potencialización de diversas habilidades, que les permiten lograr objetivos y metas desde los intereses o deseos individuales de desarrollo (Daza, Castañeda, Tovar, Segovia, Cortés, 2019). Por tanto, cobra sentido comprender al estudiante como un agregado de dimensiones; que deben fortalecerse desde el proceso educativo, asumiendo desde la práctica docente la responsabilidad de plantear estrategias pedagógicodidácticas que privilegien el desarrollo de cada una de 
ellas, entendiendo que estas son acciones que contribuyen al logro de los objetivos de aprendizaje y transforman la conducta humana en favor de la formación en valores tal como lo plasman (Guarnizo, Talero y Campos, 2021;Nova 2016, citado por; Villegas, Alderrama y Suárez 2019). De acuerdo con lo anterior, es posible afirmar que toda acción educativa dirigida a los educandos propende por su desarrollo multidimensional, vinculando cada uno de los elementos que le componen como ser humano único.

Asimismo, la formación integral contribuye a enriquecer los procesos de socialización del estudiante, afinando su sensibilidad, mediante el desarrollo de todas sus facultades y contribuyendo a su desarrollo moral, espiritual y crítico (Fichte, 1977; citado por Orozco, 2008). De ahí la importancia de formar integralmente a los estudiantes en la escuela y generar estrategias para tal fin. Así pues, «la institución educativa, en su reto y tarea diaria de educar y favorecer el desarrollo integral de las personas, ha obviado al cuerpo y muchas de sus posibilidades» (Águila y López, 2018,p.413), por ello es tan importante aprovechar un área como la educación física para favorecer el desarrollo. Entre tanto, (Guerra, Mórtigo y Berdugo, 2014; Orozco, 2008; Yepes y Reyes, 2014) coinciden en afirmar que una formación completa desarrolla todas las potencialidades del ser humano, considerándolo no solamente desde un enfoque cognitivo o de saberes específicos, sino también ofreciendo los elementos necesarios para que crezca como persona y busque una plena realización mejorando su calidad de vida y la del entorno en el que se desarrolla.

Ahora bien, Villegas et al., (2019) afirman que la formación integral reconoce las facetas del estudiante, y fomenta su convivencia con el entorno en congruencia con una personalidad reflexiva, crítica, sensible, creativa y responsable, tocando las dimensiones que como sujeto social le son inherentes. Para el estudiante, su desarrollo pasa por la construcción de conocimientos integrados, experiencias sociales, idoneidad para la comunicación argumentativa, así como facultades reflexivas, analíticas, críticas y valores éticos de respeto, responsabilidad y consideración por el medio ambiente (Camarena, 2014; citado por Daza et al., 2019). La formación integral va más allá de la simple adquisición de conocimientos, se trata de cómo el estudiante se construye a partir de ellos y logra transformar su entorno, convirtiéndose en un ciudadano que haga de su contexto un mejor espacio. En este sentido, «la formación integral entonces es entendida como la formación de todos los aspectos que conforman el ser humano, teniendo en cuenta la complejidad que ello implica.»
(Pinilla, Gamboa y Patiño 2021,p. 148).

Complementando, Acodesi (2005) al igual que,Yepes y Reyes (2014) proponen, que el ser humano está integrado por las dimensiones ética, espiritual, cognitiva, afectiva, comunicativa, estética, corporal y sociopolítica, a través de ellas, se debe desarrollar su proceso educativo para convertirlo en integro, preparándolo para un vida plena y armónica en la que pueda desempeñarse y vivir en comunidad. Si bien es cierto que estos factores son esenciales en el proceso educativo, no se puede ser ajeno a unas nuevas perspectivas en las que el ser humano definitivamente requiere ser formado, ya que las mismas condiciones actuales han contribuido para que en especial la juventud esté más conectada a las nuevas tecnologías, pero menos conectada con los otros seres humanos que los rodean físicamente y muchísimo menos conectado con sus propios sentimientos y emociones (Guerra et al., 2014).

«La aparición de nuevas tecnologías y más específicamente de las redes sociales interactivas cambiaron la manera de conectar el mundo, las comunicaciones sin importar las distancias se hicieron más eficaces en cuanto a tiempo y calidad, pero tal vez el avance más significativo recae en la aparición de la red, que en un modo más simple de entender es el internet, facilitó la forma de acceder a información, gracias a que a través del mismo se puede conseguir cualquier información que se requiera con tan solo un clic y en lapsos cortos de tiempo» (Bernate, Fonseca, Guataquira y Perilla 2021, p. 311). De acuerdo a lo anterior, es posible y necesario pensar en una dimensión tecnológica, en la que se forme al estudiante para el uso adecuado tanto de los medios tecnológicos actuales, como de las herramientas que la misma sociedad le ha proporcionado; y no menos importante en una dimensión socioemocional en la que el ser humano pueda estar conectado con sus sentimientos y emociones, en la que además desarrolle habilidades y competencias que le permitan manejar de forma asertiva situaciones que le puedan afectar.

Por otra parte, la formación de los estudiantes se consolida cuando se enfrentan a situaciones problémicas que les hacen reflexionar, cuestionar y actuar (Reynoso y González, 2018), para ello es necesario plantear estrategias que involucren "la motivación espontánea de la persona, se debe fomentar la creatividad, el autoconocimiento, y el respeto de su valor como ser humano, sin pasar por encima de los demás» (Betancourt, Bernate, Fonseca, y Rodríguez 2020,p. 848). De ahí, la importancia de generar estrategias o actividades pedagógico-didácticas como salidas de campo, juegos tradicionales (recordando al abuelo), escuela de formación deportiva (semillero Isidorista), juegos 
intercursos (mi recreo), campañas (me alimentó y me cuido bien), cine foros, unidades didácticas específicas, expresa lo que piensas, uso de redes sociales y carreras de observación con apoyo de herramientas tecnológicas (App Munzee), aplicadas en esta investigación, que permitan el desarrollo de los procesos de formación, evaluar la incidencia de las mismas y generar condiciones de calidad en el aprendizaje, tal como lo proponen Pinilla, et al. (2021), al implementar estrategias que vinculan el componente tecnológico en la clase de educación física para contribuir en los aprendizajes significativos y la percepción de la formación integral de los estudiantes.

Ahora bien, el ser humano interactúa con el mundo a través de su corporeidad, la cual es protagonista de todo acto humano, visible o invisible, permitiendo generar nuevas experiencias (Trigo, 2000; citado por Águila y López, 2018). En este sentido, la educación física desde la motricidad desarrolla en el ser humano una variedad de experiencias significativas, $\mathrm{Al}$ respecto, Miñana y Monfort (2020) manifiestan que debe ser entendida como una acción educativa que atañe a la relación consigo mismo, con otras personas y con el mundo que le rodea, se puede definir como la disciplina pedagógica que se ocupa de la educación integral del individuo a través de la acción motriz. "La educación física se reconoce como una de las disciplinas con mayor importancia y es fundamental en la formación de cada uno de los individuos que interactúan en la sociedad, se puede ver de manera exitosa si esta se implementa a una corta edad, debido al desarrollo de habilidades, destrezas motoras, cognitivas y afectivas que son esenciales para la vida.» (Betancourt, et al., p. 848). Añadiendo, García (2016) sostiene que la educación física posibilita la construcción del ser humano con formación integral y competencias para afrontar las nuevas demandas sociales.

Asimismo, la educación física orienta las posibilidades de desarrollo de capacidades cognitivas, relacionales, emocionales y expresivas del movimiento (García, 2016). En este sentido, Águila y López, (2018) afirman que se nace con un cuerpo que se transforma, se adapta y conforma una corporeidad a través de la experiencia, incluyendo la acción, las emociones, los pensamientos y la percepción sensorial. Por tanto, el desarrollo de capacidades, habilidades y la adquisición de esas experiencias, no sería posible sin la tutoría del docente, tal como lo manifiesta Martínez (2009), puesto que finalmente es quien guía, acompaña, propone y evalúa las estrategias.

Igualmente, desde el punto de vista biológico, Griban et al., (2019) argumentan que la educación física afecta las características funcionales de la actividad del sistema nervioso como base de demostraciones específicas de temperamento y a través de ella, se pueden desarrollar la fuerza, la velocidad de los procesos mentales y excitación emocional, entre otros. Este proceso se desarrolla desde el área con el fin de generar cambios conductuales en los seres humanos y ocurre exitosamente cuando son jóvenes, debido al estadio mental en el que se encuentra un adolescente. Por otro lado, la actividad física ayuda a reducir la grasa corporal, reduce los niveles de triglicéridos, reduce la presión sanguínea, mejora capacidades físicas, posee efectos positivos sobre los síntomas de ansiedad y depresión, sobre el auto-concepto físico, la concentración, la memoria, el comportamiento en clase y las relaciones con los compañeros (Programa Perseo, 2007; citado por López et al., 2016).

Resulta entonces, que la educación física es una disciplina privilegiada que no solo permite transformar el cuerpo en su exterior, sino que, como lo plantean Brasó y Torrebadella (2018), se puede contribuir a una formación crítica, no discriminada, valorando el entorno y siendo ecológico, buscando una educación para la paz, aprendiendo a vivir en y para la comunidad. En otras palabras, formado al ser humano en todas sus dimensiones, con la capacidad de expresarse, desarrollar todo su potencial desde y a través de la motricidad, como lo expresan Griban et al., (2019) con autorregulación de la psico-emocionalidad, autoestima y experiencias significativas positivas desde la actividad física.

De acuerdo con lo expuesto anteriormente, el presente artículo dará respuesta a la siguiente pregunta: ¿Qué incidencia tienen las estrategias pedagógicodidácticas implementadas desde el área de educación física sobre las dimensiones de la formación integral de los estudiantes de media vocacional?

\section{Metodología}

Para el desarrollo de esta investigación se empleó un método cuasiexperimental, el cual es útil para estudiar problemas en los cuales no se puede tener control absoluto de las situaciones, pero se pretende tener el mayor control posible, aun cuando se estén usando grupos ya formados (Segura, 2003; citado por Manterola y Otzen, 2015). Al respecto, Echevarría (2016) sostiene que son más fáciles de implementar porque se consideran grupos naturales, es decir, los toman tal como están constituidos y se asignan a los distintos tratamientos. 
Se empleó un instrumento, pretest y postest -tal como lo propone Echevarría (2016)- tipo escala Likert, previamente validado por expertos y estadísticamente confiable a través del método alfa de Cronbach para evaluar consistencia interna, cuyo resultado fue de 0.92 y un análisis de correlación entre las preguntas, el cual se compone de 56 ítems, encaminados a evaluar la percepción de desarrollo de cada una de las dimensiones desde el área de educación física, la tabla 1 muestra un aparte del instrumento aplicado el cual indago sobre la dimensión comunicativa.

Tabla 1.

Aparte instrumento

Dimensión comunicativa

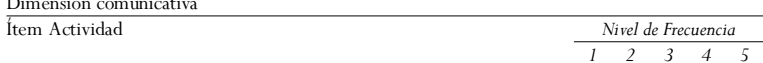

6.1 Usa el diálogo para resolver conflictos.

6.2 Utiliza el lenguaje de manera respetuosa cuando discute o se expresa.

6.3 Respeta cuando alguien está expresando su opinión.

6.4 Conserva actitudes optimistas ante las dificultades.

6.5 Acepta las críticas como medio para mejorar.

6.6 Incide fácilmente en la toma de decisiones de un grupo.

6.7 Se expresa claramente de forma convincente, a través de lenguajes o medios gestuales, verbales o gráficos.

Para este caso, la muestra estuvo compuesta por 180 estudiantes de media vocacional (últimos dos años de educación secundaria), de una institución educativa pública, con edades entre los 14 y 18 años de edad, de ambos sexos, los menores de edad participaron con la aceptación de los padres de familia de los estudiantes, quienes firmaron un consentimiento informado autorizando el tratamiento de datos personales, tal como lo dispone la legislación colombiana a través de la Ley 1581 de 2012. La muestra es significativa debido a que la población de los dos últimos años escolares de bachillerato en la institución es de 290 estudiantes, de los cuales se eligen el $62 \%$ para el desarrollo de la investigación a conveniencia del investigador, ya que un $24 \%$ no tenía ningún tipo de contacto con el interventor, por lo cual fue un motivo de exclusión de la investigación, y el $14 \%$ restante decidió de manera voluntaria no participar de la investigación o se retiró durante el proceso.

En la aplicación, el pretest se usó como diagnóstico de percepción sobre el grado de formación integral que obtuvieron desde el área de educación física; posteriormente se realizó una intervención en la que se emplearon estrategias pedagógico-didácticas como juegos tradicionales, expresa lo que piensas, recreonatación, práctica en gimnasio y Munzee, además de las unidades didácticas propias del área, que se encaminaron a fortalecer los procesos de formación de los estudiantes en coherencia con lo plateado por Pinilla et al, (2021), durante dos años y luego se aplicó un postest con el que se comparó la percepción con relación al pretest. Con respecto a los diseños cuasiexperimentales White y Sabarwal (2014), plantean que, son considerados como una intervención en la que se comprueba en qué medida un tratamiento o estrategia logra sus objetivos. En otras palabras, la comparación se realizó entre lo que ocurre antes y después de la implementación de las estrategias, para determinar la incidencia de estas en el proceso de formación integral desde la perspectiva de los estudiantes.

Finalmente, se hizo una prueba de comparación que permitió afirmar que existen diferencias estadísticamente significativas entre el grupo de estudiantes antes y después de la intervención pedagógico-didáctica. Ahora bien, dado que la distribución de las diferencias entre los promedios del pretest y del postest no presentaron una distribución normal, los datos son cuantitativos ordinales y considerando también el tamaño de la muestra, se determinó que la prueba de comparación no paramétrica más apropiada para este estudio era la prueba con rangos deWilcoxon.

En este sentido y como hallazgo, en cada una de las dimensiones se planteó como hipótesis alterna que existen diferencias significativas entre las concepciones de los sujetos sobre su formación integral antes y después de la intervención pedagógico-didáctica y se realizó la prueba de hipótesis con un nivel de significancia del 5\%.

\section{Resultados}

Para analizar la dispersión y la simetría de los datos que obtuvieron los estudiantes en las pruebas pretest y postest, que indagaron sobre el aporte de clase de educación física a las dimensiones de la formación integral, se implementaron los diagramas de cajas y bigotes. En la figura 1, se presentan los resultados del grupo de estudio y en la tabla 2, se reportan los valores por cuartiles para cada una de las dimensiones.

\begin{tabular}{|c|c|c|c|c|c|}
\hline \multirow[b]{2}{*}{ Dimensiones } & \multirow[b]{2}{*}{ Mínimo } & \multicolumn{3}{|c|}{ Cuartil } & \multirow[b]{2}{*}{ Máximo } \\
\hline & & 1 & 2 & 3 & \\
\hline E1 Ética & 1,86 & 2,7143 & 3,5000 & 4,0000 & 4,71 \\
\hline E2 Ética & 3,43 & 4,1429 & 4,2857 & 4,5357 & 5,00 \\
\hline E1 Espiritual & 1,00 & 2,5714 & 3,2857 & 4,4286 & 5,00 \\
\hline E2 Espiritual & 2,57 & 3,5714 & 3,8571 & 4,1429 & 5,00 \\
\hline E1 Intelectual & 1,00 & 2,4286 & 3,0000 & 4,1429 & 5,00 \\
\hline E2 Intelectual & 2,29 & 3,4286 & 3,7143 & 4,1429 & 5,00 \\
\hline E1 Corporal & 1,43 & 2,8571 & 3,4286 & 4,2857 & 5,00 \\
\hline E2 Corporal & 2,71 & 3,8571 & 4,0000 & 4,4286 & 5,00 \\
\hline E1 Social & 1,00 & 2,4286 & 3,2857 & 4,3929 & 5,00 \\
\hline E2 Social & 1,71 & 3,4643 & 4,1429 & 4,4286 & 5,00 \\
\hline E1 Comunicativa & 1,00 & 2,3214 & 3,2857 & 4,2857 & 5,00 \\
\hline E2 Comunicativa & 2,43 & 3,2857 & 3,5714 & 3,8571 & 4,71 \\
\hline E1 Tecnológica & 1,00 & 2,4643 & 3,1429 & 4,1429 & 5,00 \\
\hline E2 Tecnológica & 2,57 & 3,2857 & 3,5714 & 4,0000 & 5,00 \\
\hline E1 Socioemocional & 2,71 & 3,7143 & 4,0000 & 4,1429 & 4,71 \\
\hline E2 Socioemocional & 2,86 & 4,1429 & 4,2857 & 4,5714 & 5,00 \\
\hline
\end{tabular}




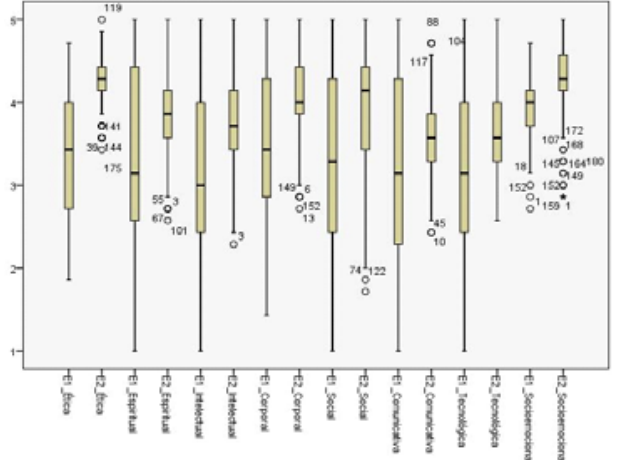

Figura 1. Resultados prueba pretest y postest sobre aportes desde la educación física a las dimensiones que constituyen la formación integral.

Los datos del pretest sobre la dimensión ética que indagó sobre las acciones y decisiones que se toman a la luz de principios y valores de manera responsable, informan que el de Q1 o cuartil inferior fue de 2,7143 puntos, y Q3 o cuartil superior de 4,0000 sobre los 5 posibles, generando un Rango Intercuartil (RI) de 1, 2857 puntos, que indica que el $50 \%$ de los datos de los estudiantes se ubicaron entre 2,7143 y 4,0000 puntos, la mediana tuvo un valor de 3.5000 y el promedio se ubicó en 3,4056 puntos. Los bigotes son ligeramente asimétricos indicando una tendencia hacia los puntajes bajos, el 25 $\%$ de los datos se ubicaron entre 1,8600 y 2,7143 puntos, la valoración más alta fue 4,7100 . El 25\% de los datos se ubicaron entre 4,0000 y 4,7100. Los datos en un 50\% estuvieron dispersos entre 2,7143 y 4,7100 puntos.

Los datos del postest en la dimensión ética indican que luego de haber participado de la estrategia pedagógico-didáctica para promoción de una formación integral, hay un incremento de 1, 4286 puntos en la mediana de los datos (Q2), ubicándose en 4,1429, por su parte, el promedio pasó de 3,4056 en el pretest a 4,2746 en el postest, la valoración más baja pasó de 1,8600 a 3,4300; se presentaron cinco datos atípicos, cuatro estudiantes con puntajes muy bajos y uno con una valoración de 5 como puntaje máximo. Se establecieron como atípicos porque están fuera del rango inferior en este caso, que correspondió a 3,4300. Los bigotes son simétricos con tendencia a puntajes altos y la simetría de la caja indica que el $50 \%$ de los datos, se ubicaron entre 4,1429 y 4,5357, la mediana pasó a ser 4,2857, indicando un aumento de 0.7857 puntos; la dispersión de los datos en menor en el postest.

Continuando con el análisis de la dimensión espiritual que preguntó sobre el reconocimiento del aporte que hacen otros para construir el sentido de su vida, la dispersión y la simetría de los datos en la prueba pretest indica que la valoración más baja fue de 1,0000 y la máxima 5, los datos están muy dispersos, en un rango de 4 puntos. El 50\% de los datos se ubicaron entre 2,5714 (Q1) y 4,4286 (Q3). Los bigotes son asimétricos, desplazándose hacia los valores bajos, el 25\% de las valoraciones estuvieron entre 2,5140 y 1,0000 . La mediana fue de 3,2857 puntos y el promedio 3,4000. Luego de la intervención, en postest, se observa que los datos se dispersan hacia puntajes más altos, el 75\% de los datos se ubicó en el rango de 2,5700 a 4,1429, la mediana se desplazó ligeramente hacia arriba, pasando de 3,2857 a 3,8571, y el marcando un incremento de 0,2857 puntos, el promedio por su parte pasó a ser 3,8389 en el postest, el puntaje mínimo pasó de 1.0000 a 2,5700, lo que indica un incremento de 1,5700 puntos. El puntaje más alto fue de 5 . La dispersión disminuyó ligeramente, se presentaron cuatro datos atípicos por debajo de 2,5700.

Siguiendo con la dimensión intelectual que cuestionó acerca de las posibilidades de hacer actividades para aprehender conceptualmente acerca de la realidad que le rodea, en el pretest se observa que los datos estuvieron altamente dispersos entre 1,0000 y 5,0000 , el 50\% de los datos estuvieron entre 2,4286 (Q1) y 4,1429 (Q3), la mediana fue de 3,000. La nota mínima en la prueba postest para la mencionada dimensión fue de 2,2900 puntos que indica un incremento de 1,2900 en el valor mínimo reportado por parte de los estudiantes, la mediana (Q2) se desplazó de 3,0000 en pretest a 3,7143 en postest, indicando una mejor valoración después de haber hecho parte de la estrategia para el fomento de la formación integral, así mismo el promedio pasó de ser 3,2286 en el pretest a 3,7389 en el postest. Luego de la intervención los datos ya no fueron tan dispersos, se ubicaron entre 2,2900 puntuación más baja hasta 5,0000 la puntuación más alta, se presentó un dato atípico hacia puntaje bajo, un estudiante valoró con menos de 2,2900.

Respecto a la valoración de los aportes a la dimensión corporal, la cual cuestionó sobre las costumbres y decisiones sobre el cuidado personal y la adquisición de habilidades para manifestarse con su cuerpo y desde su cuerpo. En pretest, los estudiantes valoraron de la siguiente manera, el 50\% de los datos entre 2,8571 (Q1) a 4,2857 (Q3), el valor mínimo fue de 1,4300 y el máximo de 5,0000, los bigotes son asimétricos con tendencia a puntajes bajos, el 25\% de los datos se ubicaron entre 2,8571 y 1,4300 puntos. La caja es asimétrica, la mediana fue de 3,4286 (Q2). Comparando los datos con el postest se evidencia un incremento en el mínimo puntaje de 1,2800 puntos, pasando de 1,4300 a 2,7100, el 75\% de los datos se ubicaron entre 3,8571 hasta 4,4286, la dispersión es menor, los datos se 
desplazaron hacia puntajes altos, la mediana (Q2) incrementó 0,5714 puntos pasando de 3,4286 a 4,0000, el promedio pasó de 3,5529 en el pretest a ser 4,0349, con lo que puede notarse un aumento de 0,4820 . Se presentaron cuatro datos atípicos, es decir, valoraciones inferiores al mínimo, por debajo de 2,7100 puntos que fue el valor inferior.

Ahora bien, con respecto a la dimensión social la cual sondeó sobre sus relaciones interpersonales y el impacto de las estrategias a nivel de convivencia, se observa que en el pretest el valor más bajo correspondió a 1,0000 y el más alto 5,0000, los datos son dispersos, el 50\% de los datos, se ubicaron entre 2,4286 (Q1) y 4,3929 (Q3), la mediana fue de 3,2857(Q2). En el postest se evidencia una mejora significativa, teniendo en cuenta que hubo un incremento de 0,8572 puntos en la mediana (Q2), variación entre Q1 de pretest y postest fue de 1,0357 puntos y la variación entre Q3 del pretest y es postest fue 0,0357 , los datos se desplazaron hacia puntajes superiores. Por su parte el promedio que en el pretest fue de 3,3706 aumentó 0,6000 puntos para resultar un promedio en el postest de 3,9706. Se presentaron dos datos atípicos de puntajes bajos, es decir dos estudiantes valoraron con menos de 1,7100 puntos sobre 5 puntos posibles.

Por otro lado, los indicadores de la dimensión comunicativa la cual indagó sobre el uso del diálogo para mejorar y aprender, de igual manera sobre el sentido y significado de la interacción lingüística con los demás, el pretest tuvo un promedio de 3,3714 mientras que en el postest fue de 3,5802. Respecto de la dispersión de los datos en el pretest se obtuvieron datos entre 1,0000 y 5,0000 , el 50\% de los datos se ubicaron entre 2,3214 (Q1) y 4,2857 (Q3), es decir, el RI fue de 1,9643. La mediana fue de 3,2857 (Q2), los bigotes son asimétricos con tendencia hacia puntajes bajos, el 25\% de los datos se ubicaron entre 2,3214 y 1,0000. Comparando con los resultados del postest, el puntaje más bajo pasó de 1,0000 a 2,4300 que indica que las valoraciones son más altas, Q1, se desplazó hacia un puntaje superior, pasando de 2,3214 a 3,2857, se logró un incremento de 0,9643. El RI disminuyó pasando de 1,9643 a 0, 5714, es decir los datos ya no son tan dispersos, el $75 \%$ de ellos se ubicaron entre 2,4300 y 3,8571, un 25\% de los datos, se ubicó entre 3,8571 y 4,7100. Se presentaron tres datos atípicos hacia puntajes altos (mayores a 4,7100 puntos) y dos atípicos hacia puntajes bajos (menores a 2,4300 puntos).

Prosiguiendo con la dimensión tecnológica la cual exploró sobre la importancia que le otorgan los estudiantes al uso adecuado de recursos TIC para su desarrollo, el aumento del promedio fue de 0,3896 pasando de 3,2921 en el pretest a 3,6817 en el postest. Por otro lado, en el pretest los estudiantes valoraron entre 1 y 5 la incidencia del uso de la tecnología en la clase de educación física, sobre la formación integral, los datos son dispersos el RI es de 1,6786, que indica que el $50 \%$ de los datos están en el rango comprendido entre 2.4643 (Q1) y 4,1429 (Q3), los bigotes son asimétricos, los datos se desplazaron hacia puntajes altos; el 25\% de las valoraciones se ubicaron entre 4,1429 y 5,0000 . Contrastando con los resultados del postest, luego de haber participado en la intervención pedagógico-didáctica, se observa que las valoraciones incrementan positivamente, el valor mínimo pasó de 1,0000 a 2,5700 puntos, es decir hubo un incremento de 1,5700 puntos hacia puntajes altos, el RI disminuyó de 1,6786 a 0,7143, es decir, los datos estuvieron menos dispersos, el 75\% de ellos, se ubicaron entre 2,5700 y 4,0000; el 25\% entre 4,0000 y 5,0000.

Para ir cerrando, los datos de la dimensión socioemocional la cual cuestionó sobre la capacidad de autorregularse y expresar sus emociones adecuadamente, se comportaron de la siguiente forma, el promedio pasó de ser 3,9239 a ser 4,2667 en el postest, frente a la dispersión de los datos puede notarse que estos son los menos dispersos tanto en pretest como en el postest, el valor mínimo en pretest fue de 2,7100 y en postest de 2.8600, se logró un incremento de 0,1500 puntos. El rango inferior (Q1) de pretest a postest incrementó 0,4286, pasando de 3,7143 a 4.1429, indicando una mejor percepción, la mediana (Q2) pasó de 4,000 a 4.2857, incrementando en 0,2857 puntos, el rango superior en pretest (Q3) pasó de 4,1429 a 4,5714, incrementando 0,4285 . El RI no varía significativamente, pasó de 0,4286 a 0,4285, lo que confirma que los datos no se dispersan tanto como en las otras dimensiones, los bigotes fueron ligeramente asimétricos hacia puntajes bajos en pretest y postest y se presentaron datos atípicos hacia puntajes bajos en las dos pruebas.

Por otra parte, las valoraciones de todas las dimensiones fueron más dispersas en pretest que en postest, lo cual es un indicativo del efecto positivo de la intervención en la clase de educación física, los datos de los postest son menos dispersos y tienen una tendencia hacia los puntajes altos. Todas las medias se desplazan hacia valores superiores a los registrados en pretest, que se constituye en otro indicador de mejora en la percepción sobre los aportes de la clase a la formación integral de los estudiantes. 
Los resultados para las pruebas de hipótesis pueden resumirse en la tabla 3 , en la que se puede notar rangos de Wilcoxon en las tres primeras columnas, además, el p-valor que en comparación con el nivel de significancia de 0.0500 permite aceptar la hipótesis nula de igualdad o rechazarla, en cuyo caso se acepta la hipótesis alterna de que existe una diferencia significativa entre los promedios de las concepciones de los sujetos sobre su formación integral antes y después de la intervención.

\begin{tabular}{|c|c|c|c|c|c|}
\hline Dimensión & Rangos positivos & Rangos negativos & Empates & $\mathrm{Z}$ & P-valor \\
\hline Ética & 153 & 15 & 12 & -10.515 & $07,335 \mathrm{E}-26$ \\
\hline Espiritual & 108 & 61 & 11 & -5.345 & $9,051 \mathrm{E}-8$ \\
\hline Intelectual & 117 & 59 & 4 & -5.771 & $7,868 \mathrm{E}-9$ \\
\hline Corporal & 109 & 63 & 8 & -5.850 & $2,398 \mathrm{E}-8$ \\
\hline Social & 112 & 60 & 8 & -5.724 & $1,041 \mathrm{E}-8$ \\
\hline Comunicativa & 97 & 73 & 10 & -2.243 & .025 \\
\hline Tecnológica & 113 & 59 & 8 & -4.420 & $1,0 \mathrm{E}-5$ \\
\hline Socioemocional & 138 & 29 & 13 & -8.425 & $3,609 \mathrm{E}-17$ \\
\hline
\end{tabular}

Respecto a los rangos de Wilcoxon puede notarse una mayor cantidad de rangos positivos en cada una de las dimensiones, rangos positivos en un mayor número de sujetos en las dimensiones Ética (153) y Socioemocional (138) que indican un aumento superior en estas dos dimensiones. Sin embargo, como se ha dicho, el aumento se presenta en todas las dimensiones, con un promedio positivo en 118 de los sujetos.

Con relación a la prueba de hipótesis, se consideró un nivel de significación usual de 0.0500 , ahora bien, dado que es posible observar que en todas y cada una de las dimensiones el p-valor es menor que 0.0500 se puede establecer con seguridad que, en efecto, existen diferencias significativas entre las concepciones antes y después de la intervención pedagógico-didáctica. Diferencias estadísticas incluso en la dimensión comunicativa en donde el p-valor de 0,0250 se acerca al nivel de significancia, y que, en todos los casos, estas diferencias, dados los rangos analizados, son positivas.

\section{Discusión}

Se observa que en todas las dimensiones hubo un aumento significativo en cuanto a la percepción de formación integral de los estudiantes, demostrando de esta manera que las estrategias implementadas a través de la intervención consideran el cuerpo no como una máquina u objeto de entrenamiento, sino como un medio para transformar seres humanos, las emociones y la afectividad tal como lo plantean Águila y López, (2018), es posible mejorar la interacción del ser humano con el mundo a través de su experiencia, permitiendo que se desarrolle plenamente.

Con relación, al aumento a un nivel superior de las dimensiones ética y socioemocional, esto puede atribuirse a implementación de las estrategias salidas de campo, juegos tradicionales, escuela de formación deportiva, juegos intercursos, campañas de estilos de vida saludables, cine foros, unidades didácticas específicas, expresa lo que piensas, uso de redes sociales y carreras de observación con apoyo de herramientas tecnológicas que fueron oportunidades para desarrollar las facultades morales, éticas y emocionales, de los estudiantes afinando su sensibilidad y por supuesto generando cambios conductuales en coherencia con lo expuesto por García (2016), Orozco (2008), Reynoso y González (2018).

Por otra parte, las diferencias estadísticas en la dimensión comunicativa en donde el p-valor de 0,025 se acerca al nivel de significancia, demuestra tal y como lo proponen Miñana y Monfort (2020) que a través de la educación física el ser humano puede interactuar con su entorno y en este sentido mejora los procesos de comunicación entre los individuos, lo cual se refleja en el resultado. Con relación al aumento de cada dimensión al revisar los resultados de comparación entre pretest y postest es válido afirmar que la educación física de acuerdo con López et al., (2016), cumple con sus finalidades en cuanto al desarrollo físico-motriz, la creación y recreación de la cultura física y su aportación al planteamiento global de desarrollo integral de los estudiantes, y por supuesto con Martínez (2009) quien establece la formación integral como proceso de los seres humanos con ayuda del docente quien le posibilita al estudiante el desarrollo de todas sus dimensiones.

Finalmente, es viable estar de acuerdo con Griban et al., (2019) con relación a la autorregulación de la psico-emocionalidad, autoestima, la influencia biológica y experiencias significativas positivas que los estudiantes reciben desde la actividad física, pues ello se hace evidente al conseguir un cambio en los temperamentos y actitudes de estos, lo cual se refleja en los resultados del postest.

\section{Conclusiones}

A través de la educación física no solamente se forma al ser humano en nuevas clases de movimiento, sino que es posible formar personas integrales capaces de desempeñarse en cualquier contexto; quedando aun lado cuestiones como el intelecto o los aspectos cognitivos relativos a la razón, como lo más importante a desarrollar en los estudiantes. Con base en la revisión de la literatura 
se incorporan en el estudio las dimensiones socioemocional y tecnológica como parte fundamental del proceso de formación integral desde el área de educación física, al ser consideradas relevantes dentro de las necesidades globales actuales partiendo de la implementación de estrategias específicas para el área.

Por lo anterior, es claro que la educación física como área es de suma importancia para la formación del ser humano en toda su integridad y que a través de ella específicamente, se promueven acciones encaminadas a potenciar dimensiones tales como la ética, espiritual, corporal, cognitiva, socioemocional, sociopolítica, tecnológica y comunicativa, generando que las estrategias empleadas salidas de campo, juegos tradicionales, escuela de formación deportiva, juegos intercursos, campañas de estilos de vida saludables, cine foros, unidades didácticas específicas, expresa lo que piensas, uso de redes sociales y carreras de observación con apoyo de herramientas tecnológicas tuvieran una gran incidencia en cuanto al proceso de formación integral, al observar cambios estadísticos significativos comparando los resultados del pretest y postest.

Lograr que el ser humano modifique conductas, es un trabajo producto de la constancia y de estrategias pedagógico-didácticas facilitadoras del proceso, y en este punto, es posible afirmar que además de las desarrolladas en esta propuesta, el papel del docente es de vital importancia, ya que en últimas es quien motiva a los estudiantes para la realización de las actividades y en gran medida la relación que se derive entre docentes y estudiantes, permitirá que ellos logren desarrollarse integralmente desde el área, o por el contrario se generen actitudes de apatía hacia la clase y su formación. Lo que permite incidir de manera positiva en la percepción sobre formación integral de los estudiantes y por supuesto evaluar su nivel, partiendo de la aplicación de un instrumento propio del área, lo cual hace parte de uno de los principales aportes de esta investigación, así como también la implementación de estrategias específicas desde el área de educación física.

Por otra parte, es importante establecer que el papel de las instituciones educativas es fundamental para desarrollar este tipo de programas de formación integral desde el área de educación física, pues solo ellas pueden fomentar modelos y procesos de enseñanza y aprendizaje que contribuyan a promover un ser humano íntegro, que piense críticamente, que se exprese de distintas formas, que se emocione, comparta, interactúe, se relacione, sea autónomo, empático y sobre todo haciendo énfasis en la relación que debe tener consigo mismo, con otras personas y el mundo que le rodea. Finalmente, se acepta la hipótesis alterna que existen diferencias significativas entre las concepciones de los sujetos sobre su formación integral antes y después de la intervención pedagógico-didáctica con una prueba de hipótesis con un nivel de significancia del 5\%, lo cual deja abierta la posibilidad de realizar investigaciones futuras con relación a la implementación de estrategias pedagógicas desde el área de educación física para contribuir con la formación integral de los educandos y además es posible proponer la realización del análisis factorial para perfeccionar un modelo dirigido la formación integral el desarrollo metodológico con base a los componentes propuestos en este estudio.

\section{Agradecimientos}

A la IE San Isidoro del Espinal en cabeza de su rector el Mg. Gilberto Carvajal Cardoso, por permitir el desarrollo de esta investigación, a la cooperación internacional de la Universidad Nacional Autónoma de México en cabeza de la Dra. Mónica Morales Barrera y al grupo AMECI reconocido y categorizado en A por MinCiencias mediante convocatoria 833 de 2018, al interior del cual me encuentro desarrollando mi trabajo de tesis doctoral.

\section{Referencias}

Águila, C., y López, J. (2018). Cuerpo, corporeidad y educación: una mirada reflexiva desde la Educación Física (Body, corporeity and education: a reflexive view from Physical Education). Retos, (35), 413-421. https:// doi.org/10.47197/retos.v0i35.62035

Almorza, D., Pérez, G., y Prada, J. (2020). An experience of comprehensive training through soccer (Una experiencia de formación integral a través del fútbol. Retos, 38, 375-378. https://doi.org/10.47197/ retos.v38i38.78566

Asociación de Colegios Jesuitas de Colombia,Acodesi (2005). La Formación Integral y sus Dimensiones:Texto Didáctico. Bogotá, Colombia: Editorial Kimpres Ltda. http:// www.acodesi.org.co/es/images/Publicaciones/ pdf_libros/texto_didactico_negro.pdf

Bernate, J., Fonseca, I., Guataquira, A., \& Perilla, A. (2020). Competencias Digitales en estudiantes de Licenciatura en Educación Física (Digital Competences in Bachelor of Physical Education students). Retos, 41, 310-318. https: / /doi.org/10.47197/retos.v0i41.85852

Betancourt, M., Bernate, J., Fonseca, I., \& Rodriguez, L. 
(2020). Revisión documental de estrategias pedagógicas utilizadas en el área de la educación física, para fortalecer las competencias ciudadanas (Documentary review of pedagogical strategies used in the area of physical education to strengthen citizen competenci. Retos, 38 , 845-851.https://doi.org/10.47197/retos.v38i38.74918

Brasó, J. y Torrebadella, X. (2018) Reflexiones para (re)formular una educación física crítica / Ideas to Re(Formulate) a Critical Physical Education. Revista Internacional de Medicina y Ciencias de la Actividad Física y el Deporte, 18(71), 441-462. http://dx.doi.org/10.15366/ rimcafd2018.71.003

Daza, J., Castañeda, J., Tovar, C., Segovia, C., y Cortés, J. (2019) Diseño y análisis psicométrico de una prueba para medir la percepción de clases frente a la formación integral de los estudiantes universitarios (PCFI). Revista ESPACIOS, 40 (2). https://www.revistaespacios.com/ a19v40n02/19400218.html

Echevarría, H. (2016). Los diseños de investigación cuantitativa en psicología y educación. Río Cuarto, Argentina: UniRio Editora. Colecciones: Académico-Científica, E-book. http://www.unirioeditora.com.ar/producto/losdisenos-investigacion-cuantitativa-psicologia-educacion/

García, S. (2016). La evaluación en Educación Física Escolar. Universidad de Tolima- Ibagué, Colombia: Editorial Kinesis

Guarnizo, N., Talero, E., \& Campos, F. (2021). Cambios de estrategias didácticas de la gimnasia en cuarentena: un estudio de caso (Changes in didactic strategies of gymnastics in quarantine: a case study). Retos, 42, 316322. https://doi.org/10.47197/retos.v42i0.86527

Griban, G., Kuzn³etsova, O., Dzenzeliuk, D., Malynskyi, I., Dikhtiarenko, Z., Yeromenko, E., Otravenko, O., Lytvynenko, A., Lyhun, N., Okhrimenko I., y Prontenko K., (2019). Dynamics of psycho-emotional state and individual psychological characteristics of students in the process of physical education classes. Dilemas Contemporáneos: Educación, Política y Valores, VII, Edición especial, 113, 1-23. https://doi.org/10.46377/ dilemas.v31i1.1326

Guerra, Y., Mórtigo, A., y Berdugo, N. (2013). Formación integral, importancia de formar pensando en todas las dimensiones del ser. Revista EducaciónY Desarrollo Social, 8(1), 48-69. https://doi.org/10.18359/reds.585

Ley Estatutaria 1581 DE 2012. Secretaría del Senado de la República, Bogotá, Colombia, 18 de octubre de 2012. http://www.secretariasenado.gov.co/senado/basedoc/ ley_1581_2012.html

López,V., Pérez, D., Manrique, J., y Monjas, R. (2016). Los retos de la Educación Física en el Siglo XXI (Challenges of Physical Education in XXI Century). Retos, (29), 182187. https://doi.org/10.47197/retos.v0i29.42552

Manterola, C., y Otzen,T., (2015). Estudios Experimentales 2 Parte: Estudios Cuasiexperimentales. International Journal of Morphology, 33(1), 382-387. https://dx.doig/ 10.4067/S0717-95022015000100060

Martínez, F. (2009). Formación integral: compromiso de todo proceso educativo. Universidad Industrial de Santander: Revista de docencia universitaria, 10(1), 123-135. https://revistas.uis.edu.co/index.php/revistadocencia/ article/view/1393

Miñana, V., y Monfort, M. (2020). Justificación del valor educativo de la Educación Física y el docente. ¿Qué profesional del deporte debe impartir la materia de Educación Física? ¿El Maestro/a y Profesor/a de Educación Física o el Monitor/a deportivo/a? (Justification of the educational. Retos, 38(38), 852-867. https://doi.org/10.47197/retos.v38i38.74722

Orozco, L. (2008). La formación integral. Mito y realidad. Revista de Ciencias Humanas y Sociales Universitas, 10161 186. https://doi.org/10.17163/uni.n10.2008.07

Pinilla, N., Gamboa, M. C.y Patiño, G. (2021). Componente tecnológico:articulación con el área educación física para promover la formación integral en la escuela. Revista Boletín Redipe, 10(7), 144-160. https://doi.org/ 10.36260/rbr.v10i7.1355

Reynoso, M., y González,V., (2018). Sustentos teóricos del diseño de la estrategia para la formación integral de Técnicos Bachiller en el Colegio Nacional de Educación Profesional Técnica, México.Didasc@lia:Didáctica y Educación, 9(4), 185-204. https:/ / dialnet.unirioja.es/ servlet/articulo?codigo $=6717870$

Villegas, F., Alderrama, C., y SuarezW. (2019). Modelo de formación integral y sus principios orientadores: caso Universidad deAntofagasta. Utopía y Praxis Latinoamericana, 24 (4), 75-88. https://www.redalyc.org/ articulo.oa:id $=279 / 27961579007$

White, H., y Sabarwal, S., (2014). Diseño y métodos cuasiexperimentales, Sintesis metodológicas: evaluación de impacto $n^{\circ} 8$, Centro de Investigaciones de UNICEF, Florencia. https: / / www.unicef-irc.org/publications/pdf/ MB8ES.pdf

Yepes, S. y Reyes, L. (2014). ¿Qué tanto saben los padres de formación integral? En Revista Educación y Humanismo, 16(26), 15-26. http:// web.a.ebscohost.com.bdigital.udistrital.edu.co:8080/ ehost $/$ pdfviewer $/$ pdfviewer?vid=1\&sid=b0e7b4446721-4488-bd57-641fdb7270a9\%40sessionmgr4008 